\title{
Identification of Unconditional Partial EfFects in Nonseparable Models
}

\author{
Christoph Rothe* \\ Toulouse School of Economics \\ This Version: July 14, 2010
}

\begin{abstract}
This note demonstrates identification of Unconditional Partial Effects introduced by Firpo, Fortin, and Lemieux (2009) in nonseparable triangular models with endogenous regressors via a control variable approach, as employed by Imbens and Newey (2009).
\end{abstract}

JEL Classification: C14, C31

Keywords: Nonseparable Models, Control Variable

*Address: Toulouse School of Economics, 21 Allée de Brienne, F-31000 Toulouse, France. Pone: +33 56112 86 78. E-mail: rothe@cict.fr. Website: http://www.christophrothe.net. 


\section{Introduction}

An important feature of many interesting economic models is that they do not imply an econometric specification with additively separable disturbance terms when they are taken to data. The properties of nonseparable econometric models have therefore received considerable interest in the recent literature, being investigated by Chesher (2003), Matzkin (2003), Chesher (2005), Chernozhukov and Hansen (2005), Hoderlein and Mammen (2007), Chernozhukov, Imbens, and Newey (2007) and Imbens and Newey (2009), amongst others. One of the most important issues in this context is how to accommodate the presence of endogenous regressors, which are frequently encountered in microeconometric applications. A possible approach is the use of so-called control variable techniques (see e.g. Blundell and Powell (2003), Blundell and Powell (2004), and Florens, Heckman, Meghir, and Vytlacil (2008)). In a recent paper, Imbens and Newey (2009) use such an approach to establish identification of various quantities of interest in triangular simultaneous equation models under relatively general conditions. These quantities include the Average Structural Function, the Quantile Structural Function, Average Derivatives and Policy Effects.

In this paper, we show that a further interesting class of parameters can be identified under general conditions in their framework: the class of Unconditional Partial Effects recently been introduced to the literature by Firpo, Fortin, and Lemieux (2009) in the exogenous case. These parameters correspond to the following thought experiment: suppose that every member of the population would experience the same exogenous marginal increase in one of its observable characteristics. How would this affect the unconditional distribution of the outcome variable? To give a concrete example, a researcher might be interested in the effect of a marginal increase in everybody's income on some feature of the distribution of consumption, such as its moments, quantiles, Gini coefficient or other measures of inequality. As pointed out by Firpo, Fortin, and Lemieux (2009), such summary measures are of interest for policy analysis, where the focus is on aggregate as opposed to individual effects of a variable.

Firpo, Fortin, and Lemieux (2009) establish that in a setting without endogenous variables, Unconditional Partial Effects are identified under weak conditions, showing that they can be represented by the average derivative of a projection of the recentered influence function of the statistic of interest on the regressors. We demonstrate that this result can be generalized to the triangular nonseparable models discussed in Imbens and Newey (2009) using their control variable approach. As a further contribution, this paper also provides a slightly different rep- 
resentation of Unconditional Partial Effects compared to the one given in Firpo, Fortin, and Lemieux (2009). We show that these parameters can be written as simple functionals of the average derivate of the conditional cumulative distribution function (CDF) of the outcome variable given the regressors and the control variable (where the derivate is taken with respect to the regressors). This representation is useful to give an explicit expression for Unconditional Partial Effects when further parametric or semiparametric restrictions are imposed on the model. This representation is by no means specific to the setting with endogenous variables but holds under full exogeneity as well, with obvious simplifications. We illustrate this point by considering the linear quantile regression model as an example.

The remainder of this paper is organized as follows. In the next section, we describe the model and give a precise definition of Unconditional Partial Effects. Identification is discussed in Section 3. The final section concludes.

\section{Model and Parameters of Interest}

The model we consider in this paper is essentially the same as in Imbens and Newey (2009). We observe a scalar outcome variable of interest denoted by $Y$, which is linked to a random vector $X=\left(X_{1}, Z_{1}\right)$ of observable determinants and an unobserved disturbance term $\varepsilon$ through the structural equation

$$
Y=g(X, \varepsilon)
$$

The subvector $X_{1}$ of $X$ is potentially endogenous and assumed to be determined through a reduced form equation,

$$
X_{1}=h(Z, \eta)
$$

where $\eta$ is another unobserved disturbance and $Z=\left(Z_{1}, Z_{2}\right)$ is a vector of instruments that exert influence on $X_{1}$ in a sense to be made precise below, but are independent of the error

terms. As in Imbens and Newey (2009), no restrictions on the dimensionality of $\varepsilon$ are imposed, allowing for general forms of unobserved heterogeneity. However, for identification purposes it will be necessary to impose such a restriction on the disturbance in $(2.2)$, as discussed below. To simplify the notation, we will focus in the following on the case with $X=X_{1}$ consisting of a single endogenous regressor only, but all arguments can easily be generalized to allow for the presence of multiple endogenous regressors or additional exogenous ones. 
The parameters we are interested in correspond to the effect of a marginal increase in $X$ on some feature $\Gamma\left(F_{Y}\right)$ of the unconditional distribution of $Y$. That is, for some constant $\delta \neq 0$, define the counterfactual random variable $Y_{\delta}$ as

$$
Y_{\delta}=g(X+\delta, \varepsilon) .
$$

Denote the CDF of $Y$ and $Y_{\delta}$ by $F_{Y}$ and $F_{Y, \delta}$, respectively, and let $\Gamma(\cdot)$ be a functional of interest. For example $\Gamma$ could be the functional that maps a CDF into one of it moments, or into its quantile function. With this notation, we can now formally define an Unconditional Partial Effect.

Definition 1 (Unconditional Partial Effect). For any functional $\Gamma: D(-\infty, \infty) \rightarrow S$, where $S$ is some normed space, the quantity

$$
\theta_{\Gamma}=\lim _{\delta \rightarrow 0} \frac{\Gamma\left(F_{Y, \delta}\right)-\Gamma\left(F_{Y}\right)}{\delta}
$$

is called the Unconditional Partial Effect of $X$ on $\Gamma\left(F_{Y}\right)$, provided that the limit in (2.3) exists.

\section{Identification}

In order to identify the Unconditional Partial Effects in models with endogeneity, we can use control variable techniques developed in Imbens and Newey (2009). Generally speaking, a control variable is an identified random vector that is able to absorb the dependence between the regressors and the unobserved disturbance term in the outcome equation (2.1), in the sense that $X$ and $\varepsilon$ will be independent conditional on the control variable. Imbens and Newey (2009) show that in the triangular model such a control variable is available under certain restrictions on the second equation. We repeat their result here for completeness.

Lemma 1 (Imbens and Newey, 2009). Suppose that $h(z, \cdot)$ is strictly increasing for all values of $z$, that $\eta$ is continuously distributed with strictly increasing $C D F$, and that $Z \perp(\varepsilon, \eta)$. Then $\varepsilon \perp X \mid V$, where $V=F_{X \mid Z}(X, Z)$.

The reason $V=F_{X \mid Z}(X, Z)$ has the properties of a control variable in our model is that the exclusive source of dependence between $X$ and $\varepsilon$ is their joint dependence on the disturbance term $\eta$ from equation $(2.2)$. However, under the conditions of Lemma $1, V$ is simply a one-to-one transformation of $\eta$, which in turn implies the result. 
The conditional independence property can be used to derive an explicit representation for $F_{Y, \delta}$. Using the structure of the model and the law of iterated expectations, we obtain that

$$
\begin{aligned}
F_{Y, \delta}(y) & =\int \operatorname{Pr}(g(X+\delta, \varepsilon) \leq y \mid X=x, V=v) d F_{X, V}(x, v) \\
& =\int \operatorname{Pr}(g(X, \varepsilon) \leq y \mid X=x+\delta, V=v) d F_{X, V}(x, v) \\
& =\int F_{Y \mid X, V}(y, x+\delta, v) d F_{X, V}(x, v) \\
& =\mathbb{E}\left(F_{Y \mid X, V}(y, X+\delta, V)\right) .
\end{aligned}
$$

This implies that the function $F_{Y, \delta}$ is identified if the support of the random vector $(X+\delta, V)$ is contained in the support of $(X, V)$. For identification of the Unconditional Partial Effect, it will be sufficient that this condition holds for small values of $\delta$ only. The role of this support condition is to ensure that there is a sufficient amount of variation in the endogenous regressors induced by the instruments. To see this, assume for a moment that $Z$ does not exert any influence on $X$. Then $V=F_{X \mid Z}(X, Z) \equiv t(X)$ is simply a transformation of the endogenous regressor. While the conditional independence condition $X \perp \varepsilon \mid V$ will still hold in this case, the joint support of $X$ and $V$ is now given by $\{(x, t(x)): x \in \operatorname{supp}(X)\}$, which is generally not a subset of $\{(x+\delta, t(x)): x \in \operatorname{supp}(X)\}$ for any $\delta \neq 0$.

In order to derive a general formula for the Unconditional Partial Effect of $X$ on $\Gamma\left(F_{Y}\right)$ for some general functional $\Gamma$, we first consider the simplest case where $\Gamma=i d$ is the identity mapping, i.e. $\Gamma(F)=F$. Then

$$
\begin{aligned}
\theta_{i d}(y) & =\lim _{\delta \rightarrow 0} \frac{F_{Y, \delta}(y)-F_{Y}(y)}{\delta} \\
& =\lim _{\delta \rightarrow 0} \frac{\mathbb{E}\left(F_{Y \mid X, V}(y, X+\delta, V)\right)-\mathbb{E}\left(F_{Y \mid X, V}(y, X, V)\right)}{\delta} \\
& =\mathbb{E}\left(\partial_{x} F_{Y \mid X, V}(y, X, V)\right)
\end{aligned}
$$

where the last equality follows by dominated convergence. The Unconditional Partial Effect of $X$ on $F_{Y}$ is thus simply the average derivative of the conditional CDF of $Y$ given $X$ and $V$, where the derivative is taken with respect to $X$. We formally state this preliminary finding in the following lemma.

Lemma 2. Suppose that the conditions of Lemma 1 hold, and that for some $c>0$ and $\delta \in$ $(-c, c)$ the support of $(X+\delta, V)$ is contained in the support of $(X, V)$. Then

$$
\theta_{i d}(\cdot)=\mathbb{E}\left(\partial_{x} F_{Y \mid X, V}(\cdot, X, V)\right)
$$

and is thus identified. 
Using the result in Lemma 2, one can now easily extend the analysis of Unconditional Partial Effects to more general quantities $\Gamma\left(F_{Y}\right)$, if $\Gamma(\cdot)$ is sufficiently "smooth". In particular, we consider functionals that satisfy a Hadamard differentiability condition, where $\Gamma$ is called Hadamard differentiable at $F$ if there exists a continuous linear functional $\Gamma_{F}^{\prime}$ such that

$$
\lim _{\delta \rightarrow 0}\left\|\frac{\Gamma\left(F+\delta h_{\delta}\right)-\Gamma(F)}{\delta}-\Gamma_{F}^{\prime}(h)\right\|=0
$$

for all sequences of function $h_{\delta} \rightarrow h$ such that $F+\delta h_{\delta}$ is contained in the domain of $\Gamma$ for some sufficiently small value of $\delta$. See van der Vaart (2000, Chapter 20.2) for further details.

To derive a general representation of Unconditional Partial Effects on $\Gamma\left(F_{Y}\right)$, define the function $h_{\delta}$ through $h_{\delta}=\left(F_{Y, \delta}-F_{Y}\right) / \delta$. We then obtain that

$$
\begin{aligned}
\theta_{\Gamma} & =\lim _{\delta \rightarrow 0} \frac{\Gamma\left(F_{Y, \delta}\right)-\Gamma\left(F_{Y}\right)}{\delta} \\
& =\lim _{\delta \rightarrow 0} \frac{\Gamma\left(F_{Y}+\delta h_{\delta}\right)-\Gamma\left(F_{Y}\right)}{\delta} \\
& =\Gamma_{F}^{\prime}\left(\theta_{i d}\right),
\end{aligned}
$$

where the last equality follows from the fact that $h_{\delta} \rightarrow \theta_{i d}$, which was shown above. That is, we can identify general Unconditional Partial Effects by using the effect of $X$ on the unconditional CDF of $Y$ as a building block. We formalize this finding in the following Theorem.

Theorem 1. Suppose that the conditions of Lemma 2 hold, and that the functional $\Gamma$ is Hadamard differentiable at $F_{Y}$ with derivative $\Gamma_{F}^{\prime}$. Then the Unconditional Partial Effect of $X$ on $\Gamma\left(F_{Y}\right)$ is given by $\theta_{\Gamma}=\Gamma_{F}^{\prime}\left(\theta_{i d}\right)$.

This representation of the Unconditional Partial Effect of $X$ on $\Gamma\left(F_{Y}\right)$ given in Theorem 1 is convenient for two reasons. First, results on Hadamard differentiability are widely available in the literature for many functionals of interest. Under appropriate conditions, this smoothness property is fulfilled for moments and quantiles, but also for inequality measures like the Gini coefficient and the Lorenz curve. See e.g. Rothe (2010) and the references therein. Second, the above representation can be useful when further parametric or semiparametric restrictions are imposed on the relationship of the outcome variable and the regressors, such as e.g. a linear quantile regression model or a single index model. In this case, the Unconditional Partial Effect of $X$ on $F_{Y}$ itself is usually still easy to compute, and results for other statistics of interest follow immediately from Theorem 1. Our representation thus allows us to establish a tight link between the Unconditional Partial Effects and the structural features of the model. This result is not specific for models with endogeneity, but applies analogously to the exogenous case where 
the control variable $V$ is not present. On the other hand, the general representation in Firpo, Fortin, and Lemieux (2009) for the exogenous case, using a projection of the recentered influence function of $\Gamma\left(F_{Y}\right)$ on the regressors, can be more difficult to evaluate for specific models.

We now illustrate this last point by considering the case where the model in equation (2.1) is a standard linear quantile regression model (see Koenker (2005)). That is, suppose that $\varepsilon$ is now a scalar random variable, normalized to be uniformly distributed on $[0,1]$, and that

$$
g(X, \varepsilon)=\beta_{1}(\varepsilon)+X \beta_{2}(\varepsilon)
$$

where $\beta_{1}(\cdot)$ and $\beta_{2}(\cdot)$ are strictly monotonic functions. The form of 2.2 can remain unchanged. Using standard arguments, one obtains that under this specification we have that

$$
\partial_{x} F_{Y \mid X V}(y, x, v)=-f_{Y \mid X V}(y, x, v) \beta_{2}\left(F_{Y \mid X V}(y, x, v)\right)
$$

and thus the Unconditional Partial Effect of $X$ on $F_{Y}$ is given by

$$
\theta_{i d}(\cdot)=-\mathbb{E}\left(f_{Y \mid X V}(\cdot, X, V) \beta_{2}\left(F_{Y \mid X V}(\cdot, X, V)\right)\right) .
$$

Now consider the Unconditional Partial Effect of $X$ on $\Gamma\left(F_{Y}\right)$, where $\Gamma(F)[\tau]=F^{-1}(\tau)=$ $\inf \{y: F(y) \geq \tau\}$ is the functional that transfers a CDF into its quantile function. Then under some standard restrictions (ensuring e.g. uniqueness of the quantiles) this map is Hadamard differentiable at $F_{Y}$ with derivative

$$
\phi \mapsto \Gamma_{F_{Y}}^{\prime}(\phi)=-\left(\frac{\phi}{\partial_{y} F_{Y}}\right) \circ F_{Y}^{-1}
$$

Writing $F_{Y}^{-1}(\tau)=q_{\tau}$ leads to the following expression for the Unconditional Partial Effect:

$$
\theta_{\Gamma}(\tau)=-\frac{\theta_{i d}\left(q_{\tau}\right)}{f_{Y}\left(q_{\tau}\right)}=\frac{\mathbb{E}\left(f_{Y \mid X V}\left(q_{\tau}, X, V\right) \beta_{2}\left(F_{Y \mid X V}\left(q_{\tau}, X, V\right)\right)\right)}{f_{Y}\left(q_{\tau}\right)} .
$$

Note that this is a weighted average of the function $\beta_{2}$ evaluated at $F_{Y \mid X V}\left(q_{\tau}, X, V\right)$, which can be interpreted as the "rank" of $q_{\tau}$ in the distribution of $Y$ conditional on $X$ and $V$. Firpo, Fortin, and Lemieux (2009) obtain a similar result for the exogenous case (compare their Proposition 1). However, while their arguments are specific for the case where $\Gamma\left(F_{Y}\right)$ is the quantile function only, our analysis can easily generalized to other statistics, such as the Lorenz curve or the Gini coefficient, via the result in Theorem 1, as long as the Hadamard differentiability condition holds.

It is also possible to derive a more general link between the Unconditional Partial Effects and structural function $g$ in equation (2.1). To make the result comparable to Proposition 1 
in Firpo, Fortin, and Lemieux (2009), suppose for simplicity that $\varepsilon$ is univariate, and that the function $g(x, \cdot)$ is strictly monotonic for all $x$, and let the function $\varepsilon_{y}(x)$ be the solution to the equation $y=g\left(x, \varepsilon_{y}(x)\right)$. Then it can be shown using arguments analogous to the ones in the proof of Proposition 1 in Firpo, Fortin, and Lemieux (2009) that

$$
\theta_{i d}(y)=-\mathbb{E}\left(\partial_{x} g\left(X, \varepsilon_{y}(X)\right) f_{Y \mid X V}(y, X, V)\right) .
$$

For the Unconditional Partial Effect of $X$ on the quantile function of $Y$ we then immediately obtain that

$$
\theta_{\Gamma}(\tau)=\frac{\mathbb{E}\left(f_{Y \mid X V}\left(q_{\tau}, X, V\right) \partial_{x} g\left(X, \varepsilon_{q_{\tau}}(X)\right)\right.}{f_{Y}\left(q_{\tau}\right)}
$$

and results for other Hadamard differentiable functionals follow directly as well.

\section{Conclusions}

In this paper, we established the identification of Unconditional Partial Effects introduced by Firpo, Fortin, and Lemieux (2009) in general nonseparable models with endogenous regressors using a control variable approach due to Imbens and Newey (2009). We also show that these effects can be written in terms of an average derivative of the conditional CDF of the outcome variable $Y$ given the regressors $X$ and the control variable $V$, where the derivative is taken with respect to $X$. This representation is useful to give an explicit expression for Unconditional Partial Effects in nonlinear parametric or semiparametric models.

\section{References}

Blundell, R., And J. Powell (2003): "Endogeneity in Nonparametric and Semiparametric Regression Models," Advances in Economics and Econometrics: Theory and Applications, Eighth World Congress, 2.

(2004): "Endogeneity in Semiparametric Binary Response Models," Review of Economic Studies, 71(3), 655-679.

Chernozhukov, V., and C. Hansen (2005): "An IV Model of Quantile Treatment Effects," Econometrica, 73(1), 245-261.

Chernozhukov, V., G. Imbens, and W. Newey (2007): "Instrumental Variable Estimation of Nonseparable Models," Journal of Econometrics, 139(1), 4-14. 
Chesher, A. (2003): "Identification in Nonseparable Models," Econometrica, 71(5), 14051441.

(2005): “Nonparametric Identification under Discrete Variation," Econometrica, 73(5), $1525-1550$.

Firpo, S., N. Fortin, And T. Lemieux (2009): "Unconditional Quantile Regressions," Econometrica, 77(3), 953-973.

Florens, J., J. Heckman, C. Meghir, and E. Vytlacil (2008): "Identification of Treatment Effects Using Control Functions in Models With Continuous, Endogenous Treatment and Heterogeneous Effects," Econometrica, 76(5), 1191-1206.

Hoderlein, S., And E. Mammen (2007): "Identification of Marginal Effects in Nonseparable Models Without Monotonicity," Econometrica, 75(5), 1513-1518.

Imbens, G., And W. Newey (2009): "Identification and Estimation of Triangular Simultaneous Equations Models Without Additivity," Econometrica, 77(5), 1481-1512.

Koenker, R. (2005): Quantile Regression. Cambridge University Press.

Matzkin, R. (2003): "Nonparametric Estimation of Nonadditive Random Functions," Econometrica, 71(5), 1339-1375.

Rothe, C. (2010): "Nonparametric Estimation of Distributional Policy Effects," Journal of Econometrics, 155(1), 56-70.

VAn Der VAart, A. (2000): Asymptotic Statistics. Cambridge University Press. 